\title{
Swallowing Disorders
}

National Institute of Neurological Disorders and Stroke (NINDS)

\section{Source}

National Institute of Neurological Disorders and Stroke (NINDS). Swallowing Disorders

Information Page.

Having trouble swallowing (dysphagia) is a symptom that accompanies a number of neurological disorders. The problem can occur at any stage of the normal swallowing process as food and liquid move from the mouth, down the back of the throat, through the esophagus and into the stomach. Difficulties can range from a total inability to swallow, to coughing or choking because the food or liquid is entering the windpipe, which is referred to as aspiration. When aspiration is frequent a person can be at risk of developing pneumonia. Food may get "stuck" in the throat or individuals may drool because they cannot swallow their saliva. Neurological conditions that can cause swallowing difficulties are: stroke (the most common cause of dysphagia); traumatic brain injury; cerebral palsy; Parkinson disease and other degenerative neurological disorders such as amyotrophic lateral sclerosis (ALS, also known as Lou Gehrig's disease), multiple sclerosis, prog ressive supranuclear palsy, Huntington disease, and myasthenia gravis. Muscular dystrophy and myotonic dystrophy are accompanied by dysphagia, which is also the cardinal symptom of oculopharyngeal muscular dystrophy, a rare, progressive genetic disorder. 